\title{
TeV gamma ray opacity in PKS 2155-304
}

\author{
G. V. Bicknell ${ }^{1}$ and S. J. Wagner ${ }^{2}$ \\ 1 Research School of Astronomy and Astrophysics, Australian National University, Cotter Rd. Weston, ACT 2611, Australia \\ e-mail: geoff@mso.anu.edu.au \\ 2 Landessternwarte Heidelberg, Königstuhl 12, 69117 Heidelberg, Germany \\ e-mail: S.Wagner@lsw.uni-heidelberg.de
}

Received 8 September 2009 / Accepted 4 November 2010

\begin{abstract}
Context. Recently observed rapid time variability in the gamma ray emission from the blazar PKS 2155-304 indicates that either the site of gamma ray emission is close to the black hole or the jet has a very high Lorentz factor. Consideration of the opacity of gamma rays close to the black hole provides additional information related to these two possibilities.

Aims. We investigate the $\mathrm{TeV}$ gamma ray opacity resulting from pair production on soft photons radiated by the black hole accretion disk, considering situations where the radiation produced by the disk is close to that predicted by the Shakura-Sunyaev model and situations where the disk radiation is much less.

Methods. We utilise expressions for the pair opacity of very high energy gamma rays developed by Gould and Schréder and Donea and Protheroe and use expressions for the disk radiation field produced by an accretion disk external to a Poynting flux dominated jet. A lower level of disk emission is modelled by using the spectrum corresponding to a lower accretion rate. We also consider changes in the shape of the gamma ray sepctrum as a flare emerges from the optically thick region.

Results. If the gravitational power resulting from accretion is dissipated by radiation in the region of the disk external to the jet, then $\mathrm{TeV}$ gamma rays are opaque out to of order 40 gravitational radii $\approx 6 \times 10^{15} \mathrm{~cm}$ from the black hole. However, the opacity is considerably reduced if the disk radiates a negligible amount of gravitational power as would be the case if there is a significant disk wind external to the jet. In either case the absence of spectral changes during observed flares excludes scenarios in which the $\mathrm{TeV}$ gamma rays are emitted even in regions of modest pair opacity.
\end{abstract}

Key words. black hole physics - accretion, accretion disks - galaxies: jets - gamma rays: theory - BL Lacertae objects: general

\section{Introduction}

TeV gamma ray observations of the blazar PKS 2155-304 have revealed rapid time variability on a scale of $\Delta t \approx$ five minutes (Aharonian et al. 2007). For a Doppler factor of $\delta$ this implies a spatial scale, $D$, in the frame of the emitting plasma given by:

$D \lesssim c \delta \Delta t \approx 9 \times 10^{13}\left(\frac{\delta}{10}\right)\left(\frac{\Delta t}{300 \mathrm{~s}}\right) \mathrm{cm}$.

With the gravitational radius $r_{\mathrm{g}}=G M / c^{2}$ one finds

$\frac{D}{r_{\mathrm{g}}} \lesssim 0.6\left(\frac{M_{\mathrm{bh}}}{10^{9} M_{\odot}}\right)^{-1}\left(\frac{\delta}{10}\right)\left(\frac{\Delta t}{300 \mathrm{~s}}\right)$

assuming a black hole mass $\approx 10^{9} M_{\odot}$ (Aharonian et al. 2007). Thus, there are two immediate possibilities: (1) if the Doppler factor is typical of that estimated for a number of other gamma ray blazars, or typical of that inferred on the VLBI scale, i.e. $\delta \approx$ 10 , then the size of the emitting region is of order the gravitational radius of the black hole. This naturally places the emission region close to the black hole. (2) If the diameter of the emitting region is of order $10^{15-16} \mathrm{~cm}$, i.e. typical of estimates based upon the variability of other blazars and modelling of their emission regions, then one requires $\delta \gtrsim 100$ if the size of the emitting region is not to be too small a fraction of the jet diameter. These broadly indicative estimates highlight the interest that is raised by the rapid variability detected in very high energy gamma ray observations of PKS 2155-304.
Gamma rays are subject to absorption by pair creation on soft photons and this process significantly influences the region from which gamma ray emission can be detected. The presence of an intense radiation field prohibits the escape of gamma rays. Therefore, it is in principle possible to constrain the emission site of the TeV emission in blazars, and in PKS 2155-304 in particular, by taking pair opacity into account.

The threshold energy $\epsilon_{\text {th }}$ of a soft photon that pair produces with a gamma ray of energy $E$ when the angle between the directions of the two photons is $\theta$, is

$\epsilon_{\mathrm{th}}=\frac{2\left(m_{\mathrm{e}} c^{2}\right)^{2}}{E}(1-\cos \theta)^{-1}$

(Gould \& Schréder 1979). The minimum threshold energy is

$\epsilon_{0}=\frac{\left(m_{\mathrm{e}} c^{2}\right)^{2}}{E} \doteq 0.26\left(\frac{E}{\mathrm{TeV}}\right)^{-1}$

for a head-on collision $(\theta=\pi)$ so that $\mathrm{TeV}$ opacity is primarily influenced by the infrared radiation field. It is well known that absorption of $\mathrm{TeV}$ gamma rays by the diffuse infrared background limits the redshift from which $\mathrm{TeV}$ emission can be detected. Here we are concerned with the infrared radiation density in the vicinity of the black hole.

Gamma ray pair opacity in AGN has been considered previously by Blandford \& Levinson (1995), who introduced the concept of the gamma-sphere and by Donea \& Protheroe (2003). Blandford \& Levinson (1995) primarily considered the effect of absorption in the EGRET energy band $(\$ 10 \mathrm{GeV})$ in which case 
the soft photon energy exceeds $\approx 30 \mathrm{eV}$. Hence, their input soft photon spectrum is dominated by hard and soft X-rays, either produced within the jet or by scattering of coronal and disk photons in the broad line region of the AGN. They adopted empirical forms for the input spectra. They also considered the cascading production of lower energy gamma rays and electron-positron pairs. Donea \& Protheroe (2003), on the other hand, were concerned with absorption in the $\mathrm{TeV}$ energy range and considered the effects of pair opacity resulting from infrared photons originating from the accretion disk, the broad line region and a possible torus. Since they considered much less energetic soft photon inputs, their disk photon spectrum was determined theoretically using a version of the "jet-disk symbiosis" model (Donea \& Biermann 1996). Both Blandford \& Levinson (1995) and Donea \& Protheroe (2003) developed models for a range of black hole masses and input spectra and consider the effect of absorption on a steady emitter of gamma rays.

In this paper we consider in detail variations in the spectral index of the gamma rays as an emitting region in the jet emerges from the optically thick region. We follow the approach of Donea \& Protheroe (2003) but primarily concentrate on the effect of disk emission on pair opacity. Our disk emission model differs from Donea \& Protheroe (2003) and is based on a Poynting flux-dominated jet with a jet radius of a few gravitational radii together with a self-consistent Shakura-Sunyaev spectrum external to the jet radius (see Sect. 3). The parameters in our calculations are most directly relevant to PKS 2155-304 but are also relevant to other gamma ray emitting jets since we consider a range of accretion rates.

\section{Gamma ray absorption in the vicinity of accretion disks}

We consider the opacity of gamma rays escaping along the jet perpendicular to the disk towards an observer in pole-on geometry, as is generally assumed for Blazars. Referring to Fig. 1, the rate of change of optical depth $\tau$ with height $z$ above the accretion disk (the inverse absorption length) is given by Eq. (8) below. We first define the symbols and terms in this expression: $E$ is the gamma ray energy, $\theta$ is the angle between the direction of the soft photon and that of the gamma ray photon, $\mu=\cos \theta$, $\mu_{\text {min, } \max }=\cos \theta_{\max }$, min where $\theta_{\max }$ min are respectively the maximum and minimum values of $\theta, \epsilon_{\min }(E)=\epsilon_{0}$ is the minimum threshold energy (see Eq. (3)), $\mathrm{d} n / \mathrm{d} \epsilon \mathrm{d} \Omega$ is the number density of soft photons per unit energy per unit solid angle. The square of the electron-positron Lorentz factor in the centre of mass frame is $s$ and $\beta=(1-1 / s)^{1 / 2}$ is the corresponding velocity; $r_{0}$ is the electron radius. The pair production cross section $\sigma_{\gamma \gamma}(\beta)$ is:

$\sigma_{\gamma \gamma}(\beta)=\frac{\pi r_{0}^{2}}{2} \bar{\sigma}_{\gamma \gamma}(\beta)$

$\bar{\sigma}_{\gamma \gamma}(\beta)=\left(1-\beta^{2}\right)\left[\left(3-\beta^{4}\right) \ln \frac{1+\beta}{1-\beta}-2 \beta\left(2-\beta^{2}\right)\right]$

$\beta=(1-1 / s)^{1 / 2} \quad s=\frac{\epsilon E}{2\left(m_{\mathrm{e}} c^{2}\right)^{2}}(1-\mu)$

(Gould \& Schréder 1979). The rate of change of optical depth with distance is:

$\frac{\mathrm{d} \tau}{\mathrm{d} z}(E, z)=2 \pi \int_{\epsilon_{\min }}^{\infty} \mathrm{d} \epsilon \int_{\mu_{\min }}^{\mu_{\max }} \frac{\mathrm{d} n}{\mathrm{~d} \epsilon \mathrm{d} \Omega}(1-\mu) \sigma_{\gamma \gamma} \mathrm{d} \mu$

(Gould \& Schréder 1979; see also Donea \& Protheroe 2003).

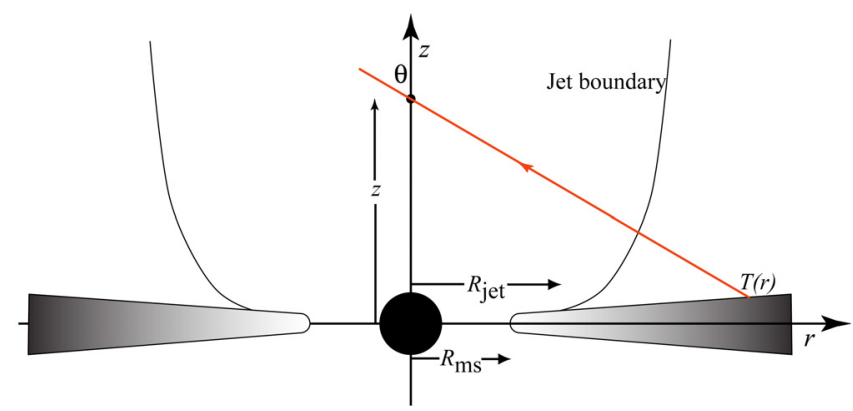

Fig. 1. The geometry of gamma ray absorption resulting from pair production in the vicinity of an accretion disk.

Approximating the accretion disk spectrum at each radius by a local black body, the number density of soft photons per unit energy per steradian is:

$\frac{\mathrm{d} n}{\mathrm{~d} \epsilon \mathrm{d} \Omega}=\frac{2 \epsilon^{2}}{h^{3} c^{3}}\left[\exp \left(\frac{\epsilon}{k T(r)}\right)-1\right]^{-1}$

where $T(r)$ is the accretion disk temperature at a radial distance $r=z \tan \theta$.

Following Gould \& Schréder (1979) we evaluate the integral (8) utilising the normalized energy variable $\eta=\epsilon / \epsilon_{0}$ (where $\epsilon_{0}$ is given by Eq. (4)) and the variable $s=s(\mu)$ defined by Eq. (7). The result is

$$
\begin{aligned}
\frac{\mathrm{d} \tau}{\mathrm{d} z}(E, z)= & \frac{2 \alpha^{2}}{\lambda_{\mathrm{e}}}\left(\frac{E}{m_{\mathrm{e}} c^{2}}\right)^{-3} \int_{\eta_{\min }}^{\infty} \mathrm{d} \eta \\
& \times \int_{s_{\min }}^{s_{\max }}\left[\exp \left(\eta \frac{\epsilon_{0}}{k T(z \tan \theta)}\right)-1\right]^{-1} s \bar{\sigma}_{\gamma \gamma} \mathrm{d} s
\end{aligned}
$$

where $\alpha$ is the fine structure constant, $\lambda_{\mathrm{e}}=h / m_{\mathrm{e}} c$ is the electron Compton wavelength, and the limits on the integrals are given by:

$\eta_{\min }=\frac{2}{1-\mu_{\min }} \quad s_{\min }=\frac{1-\mu_{\max }}{2} \eta \quad s_{\max }=\frac{1-\mu_{\min }}{2} \eta$.

Once $\mathrm{d} \tau / \mathrm{d} z$ has been numerically evaluated, the optical depth between any two points along the $z$ axis may be evaluated by a further numerical integration with respect to $z$.

Relativistic leptons generated via pair-absorption upscatter ambient radiation up to gamma-ray energies resulting in a second-order contribution to the observed gamma-ray spectra. This contribution is negligible for the considerations in this study and is not taken into account here.

\section{Spectral energy distribution of soft disk photons}

An accretion disk model is required in order to determine the temperature appearing in Eq. (10). The standard model is the Shakura \& Sunyaev (1973) model in which the gravitational power is entirely dissipated in the disk and appears as (approximately) black body radiation from the surface. However, the mechanical power in winds and jets reduces the flux of radiation from the surface (Kuncic \& Bicknell 2004). Moreover, in the case of AGN some of the gravitational power not channeled through outflows is not dissipated in the disk but in the corona in a way that is not currently understood, notwithstanding the insights provided by partly phenomenological models (Haardt \& Maraschi 1991, 1993; Haardt et al. 1994). In view of our imperfect understanding of the physics of coronal emission, it is 
not clear for blazars such as PKS 2155-304, how much of the gravitational power is channelled into disk and coronal emission, respectively.

In view of the uncertainties in the role of coronal emission and hence in the accretion disk spectral energy distribution we ignore potential dissipation in the corona and adopt the following approach:

1. We assume that the base of the jet occupies a radial range between the minimally stable circular orbit $r_{\mathrm{ms}}$ and the outer jet radius $r_{\text {jet }}$. We adopt the limiting case (Bicknell \& Li 2007) in which all of the angular momentum transported inside $r=r_{\text {jet }}$ is transported vertically by a Poynting fluxdominated jet. Consistent with this assumption, most of the accretion power liberated between $r_{\text {jet }}$ and $r_{\mathrm{ms}}$ is channeled into the kinetic power of two jets and most of the accreted mass goes into the black hole. In this limit, the power of each jet and the accretion rate, $M_{\mathrm{a}}$, are related by the following equation (for a Newtonian accretion disk; see Bicknell \& Li 2007):

$P_{\text {jet }} \approx \frac{\dot{M}_{\mathrm{a}} c^{2}}{4}\left[\left(\frac{r_{\mathrm{ms}}}{r_{\mathrm{g}}}\right)^{-1}-\left(\frac{r_{\mathrm{jet}}}{r_{\mathrm{g}}}\right)^{-1}\right]$.

In the models presented below we estimate the gamma ray opacity for specific values of the parameters $r_{\mathrm{ms}}$ and $r_{\text {jet }}$; we discuss the effect of varying these parameters further in Sect. 8.

2. Equation (12) may be used to determine the mass accretion rate given the jet power. We adopt a fiducial power $\approx 10^{44} \mathrm{erg} \mathrm{s}^{-1}$ as implied by the analysis of Begelman et al. (2008). Adopting a black hole mass $M \approx 10^{9} M_{\odot}$ for PKS 2155-304 (Aharonian et al. 2007), an Eddington luminosity, $L_{\mathrm{Edd}} \approx 1.3 \times 10^{47} \mathrm{erg} \mathrm{s}^{-1}$ and defining the normalized accretion rate $\dot{m}=\dot{M}_{\mathrm{a}} c^{2} / L_{\text {Edd }}$ gives:

$$
\begin{aligned}
\dot{m} & =\frac{4 P_{\text {jet }}}{L_{\mathrm{Edd}}}\left(\frac{r_{\mathrm{ms}}}{r_{\mathrm{g}}}\right)\left[1-\frac{r_{\mathrm{ms}}}{r_{\text {jet }}}\right]^{-1} \\
& \approx 3.2 \times 10^{-3}\left(\frac{P_{\text {jet }}}{10^{44} \mathrm{erg} \mathrm{s}^{-1}}\right)\left(\frac{r_{\mathrm{ms}}}{r_{\mathrm{g}}}\right)\left[1-\frac{r_{\mathrm{ms}}}{r_{\mathrm{jet}}}\right]^{-1} .
\end{aligned}
$$

Thus, for this Newtonian accretion disk model, we estimate $\dot{m} \approx 10^{-2}$ for $r_{\mathrm{ms}} / r_{\mathrm{g}}=2$ and $r_{\mathrm{jet}} / r_{\mathrm{g}}=5$.

3. Previous models of PKS 2155-304 (e.g. Aharonian et al. 2005) imply a lower jet power $\approx 10^{43} \mathrm{erg} \mathrm{s}^{-1}$ so that we also consider lower values of $\dot{m}$ in our models.

4. If, in the region of the disk external to the jet, there is no energetically significant wind, then the accretion disk temperature for $r>r_{\text {jet }}$ is given by a Shakura-Sunyaev model in which the turbulent stress is zero at $r=r_{\text {jet }}$. Hence, the photon number density per unit energy per unit solid angle is as given by Eq. (9) with the accretion disk temperature for $r>r_{\text {jet }}$ given by:

$$
T(r)=\left(\frac{3 m_{\mathrm{p}} c^{3}}{\sigma \sigma_{\mathrm{T}} r_{\mathrm{g}}}\right)^{1 / 4} \dot{m}^{1 / 4}\left(\frac{r}{r_{\mathrm{g}}}\right)^{-3 / 4}\left[1-\left(\frac{r_{\mathrm{jet}}}{r}\right)^{1 / 2}\right]^{1 / 4} r>r_{\mathrm{jet}}
$$

where $\sigma$ is the Stefan-Boltzmann constant.

5. As well as allowing for lower jet powers, models with lower $\dot{m}$ give us some insight into the situation where there is an energetically significant wind, which reduces the radiative flux from the disk (Kuncic \& Bicknell 2004). We therefore consider a range of values $10^{-5} \leq \dot{m} \leq 10^{-2}$.
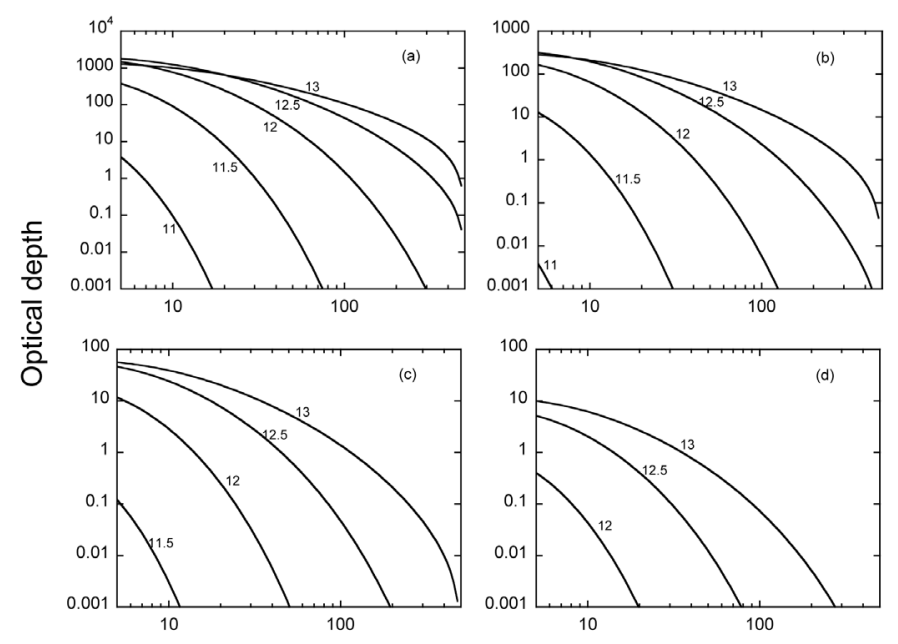

Height above accretion disk in gravitational radii

Fig. 2. The pair opacity of gamma rays for different energies and different values of the normalised accretion $\dot{m}=\dot{M}_{\mathrm{a}} c^{2} / L_{\mathrm{Edd}}$. The individual curves are labelled by the logarithm of the energy in $\mathrm{eV}$. The different panels correspond to different normalized mass accretion rates. a): $\dot{m}=10^{-2}$; b): $\left.\dot{m}=10^{-3} ; \mathbf{c}\right): \dot{m}=10^{-4}$; d) $\dot{m}=10^{-5}$.

6. We neglect gravitational effects on the energy of photons and their trajectories close to the black hole. This is discussed further below in Sect. 7

In this model the ratio of the disk power to total power is $r_{\mathrm{ms}} / r_{\text {jet }}$. Hence, for jet radii of a few gravitational radii the radiation field implied by Eq. (14) may be a factor of a few lower in intensity than expected from the standard Shakura \& Sunyaev (1973) model. Nevertheless, it is not realistic to expect that the radiative intensity is $2-3$ orders of magnitude less than that from a Shakura-Sunyaev disk on the basis of the kinetic luminosity of the jet alone. The most direct way in which the disk radiative intensity could be reduced would be if most of the gravitational power outside the jet were to be channeled into a slower disk wind. (See Sect. 4.)

The above ansatz for calculating the accretion disk spectrum differs from Donea \& Protheroe (2003) who based their calculation on a non-magnetic disk and jet model ("jet symbiosis model") in which the flux of angular momentum is proportional to the jet mass flux. This aspect is essential since there is no magnetic field to transport angular momentum. The mass flux in their model is small in order that the jet have a high asymptotic velocity. Hence, little angular momentum is transported by the jet and their disk model is close to the Shakura-Sunyaev model. In our model there is a relative deficit of radiation from the region of the disk interior to $r_{\text {jet }}$, as illustrated in Fig. 4.

\section{Estimation of opacity}

Consider first the case of an accretion disk corresponding to a normalized accretion rate $\dot{m}=10^{-2}$. The gamma ray opacity between a height $z$ above the accretion disk and 500 gravitational radii is shown in the panel (a) of Fig. 2 for a number of different energies below and above a $\mathrm{TeV}$. For $\mathrm{TeV}$ gamma rays the region is optically thick out to $\approx 100$ gravitational radii $\approx 1.5 \times 10^{16} \mathrm{~cm}$. Moreover the height of the gammasphere increases with energy; 
$10 \mathrm{TeV}$ emission remains optically thick out to approximately 500 gravitational radii.

The optical depths for the lower jet power of $10^{43} \mathrm{erg} \mathrm{s}^{-1}$ corresponding to $\dot{m} \approx 10^{-3}$ (panel (b) of Fig. 2, display a similar trend, although there is a noticeable decrease in optical depth. At a $\mathrm{TeV}$, for example, the emission starts to become optically thin at around 30 gravitational radii $\approx 4.5 \times 10^{15} \mathrm{~cm}$.

The most direct way to decrease the opacity is to reduce the level of disk radiation. This occurs if there is a disk wind in the region outside the jet thereby reducing the gravitational power that is dissipated in disk radiation. The details depend on the disk spectrum, which in turn depends on the dependence of wind mass-loss rate on radius. Here, we obtain a semi-quantitative estimate of the reduction of the opacity by simply reducing $\dot{m}$. Thus, the lower two panels of Fig. 2 indicate the opacities corresponding to $\dot{m}=10^{-4}$ and $10^{-5}$. Nevertheless, for $\dot{m}=10^{-5}$ the $10 \mathrm{TeV}$ emission is still optically thick out to about 40 gravitational radii $\approx 6 \times 10^{15} \mathrm{~cm}$.

Note that for the two highest accretion rates, the $10^{12} \mathrm{eV}$ and $10^{12.5} \mathrm{eV}$ opacity curves cross at a height of about $20 r_{\mathrm{g}}$. This occurs for the following reasons: at small heights above the accretion disk, the photon field is dominated by the inner part of the accretion disk. For the higher accretion rates the threshold energy for disk photons that pair produce with $10^{12.5} \mathrm{eV}$ gamma rays is considerably less than the mean energy of photons emitted from that region of the disk. The decrease of the pair cross section with energy means that these disk photons are less effective in producing pairs than a comparable number density of lower energy photons. Moreover the threshold energies of photons that pair-produce with $10^{12} \mathrm{eV}$ photons are closer to the mean disk photon energy. This is why their opacity in this region is slightly larger. As the emission height increases a larger region of the disk contributes to the opacity and the effect of the energetic photons from the inner disk is minimised. This effect disappears at the lower accretion rates since the inner disk is not as hot for those photons.

\section{Spectral variations}

The intrinsic spectrum is likely to exhibit a cutoff at high energies. Hence, it is not easy to unambiguously identify pairabsorption in single epoch spectra even for significant opacities.

Figure 2 shows that at a given energy the opacity is dependent on the height above the accretion disk. Therefore, a gammaray emitting region emerging from an optically thick region becomes optically thin at progressively higher energies. That is, its spectral index changes with time. For an emitting region that is close enough to the central black hole at the onset of a flare to be tranversing the regime of moderate absorption while the flare is ongoing, such spectral changes can be observed. We consider the case of the flares in PKS 2155-304 reported by (Aharonian et al. 2007). No spectral changes were observed during the $120 \mathrm{~min}$ of continuous observations.

In order to indicate how the spectrum may evolve observationally, we proceed as follows: let $N_{\text {obs }}\left(E, z_{0}\right)$ (in erg $\mathrm{cm}^{-2} \mathrm{~s}^{-1} \mathrm{erg}^{-1}$ ) be the observed spectrum at a height $z_{0}$ above which absorption is negligible, $N_{\mathrm{abs}}(E, z)$ be the observed spectrum at a lower height $z$ and $\Delta \tau\left(E, z, z_{0}\right)$ the difference in optical depth between the two heights. Then, if the intrinsic spectrum of the flare does not change during the time of propagation from $z$ to $z_{0}$,

$N_{\mathrm{abs}}(E, z)=N_{\mathrm{obs}}\left(E, z_{0}\right) \exp [-\Delta \tau(E, z, z 0)]$.

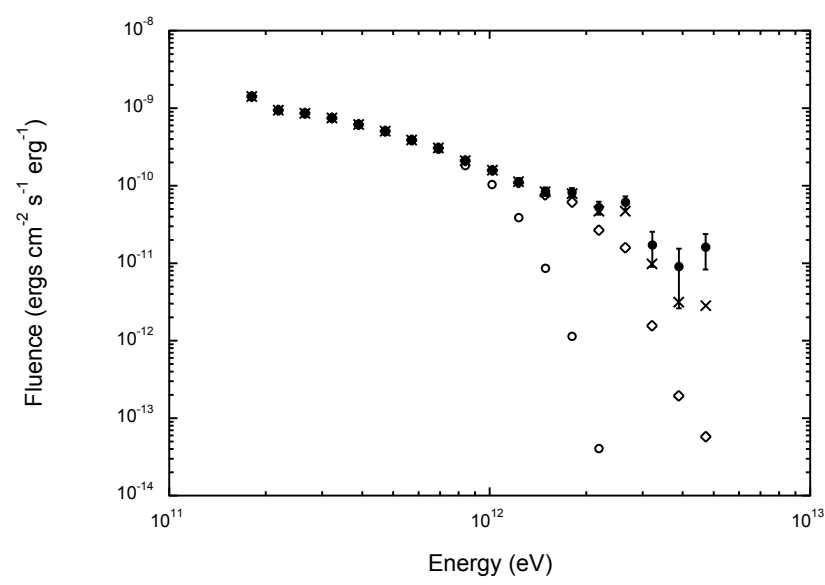

Fig. 3. The observed high energy gamma ray spectrum of PKS 2155304 modified by the optical depth at three different heights, assuming for illustration that the observed spectrum is emitted at a height of $300 r_{\mathrm{g}}$ above the accretion disk. The filled circles represent the observed spectrum. The various symbols represent the absorbed spectrum at the following heights: open circles $z=50 r_{\mathrm{g}}$; Diamonds: $z=100 r_{\mathrm{g}}$; Crosses: $z=150 r_{\mathrm{g}}$.

The differential optical depth between the two heights is given by

$\Delta \tau\left(E, z, z_{0}\right)=\tau(E, z)-\tau\left(E, z_{0}\right)=\int_{z}^{z_{0}} \frac{\mathrm{d} \tau}{\mathrm{d} z} \mathrm{~d} z$

and $\mathrm{d} \tau / \mathrm{d} z$ is given by Eq. (10).

By way of illustration we select the optical depth derived for the normalised accretion rate $\dot{m}=10^{-3}$. The optical depth is unity at $10 \mathrm{TeV}$ for a height $z=304.3$ so that the spectrum of a flare observed at this height could be assumed to be close to intrinsic. Thus, we take $z_{0}=300 r_{\mathrm{g}}$ and evaluate the absorbed spectrum according to Eq. (15) for heights $z=50,100$ and $150 r_{\mathrm{g}}$. The template spectrum is the integrated spectrum presented by (Aharonian et al. 2007), chosen to indicate realistic error bars and deviations from a power-law slope. The results of applying the relevant differential optical depths to the observed spectrum are shown in Fig. 3. As can be seen in Fig. 3 the major temporal changes in the spectrum occur for energies greater than a TeV and naturally the greatest changes are observed for the radiation emitted from closest to the black hole. The observed time for a flare to propagate from $z=50 r_{\mathrm{g}}$ to $100 r_{\mathrm{g}}$ is $2.9 / \delta$ days. The smallest changes in the observed spectrum in this example result from the propagation from 150 to $300 r_{\mathrm{g}}$; this would take $8.65 / \delta$ days. Therefore, the absence of an observable spectral change rules out stratified absorption if the flare propagates without much change in its intrinsic spectrum over this period. It would be contrived if intrinsic spectral changes would compensate the spectral variations resulting from varying amounts of pair-absorption.

\section{Discussion of some physical aspects of the models}

It is instructive to compare our disk model with the ShakuraSunyaev model by comparing the threshold energy for absorption by photons emitted from a given disk radius with the mean energy emitted at that radius. Since the threshold energy depends inversely on the angle between the two photons (see Eq. (3)), it increases as the radius from which the low energy photons are emitted, decreases. Figure 4 shows the comparison of mean emitted energy with threshold energy as a function of disk radius 


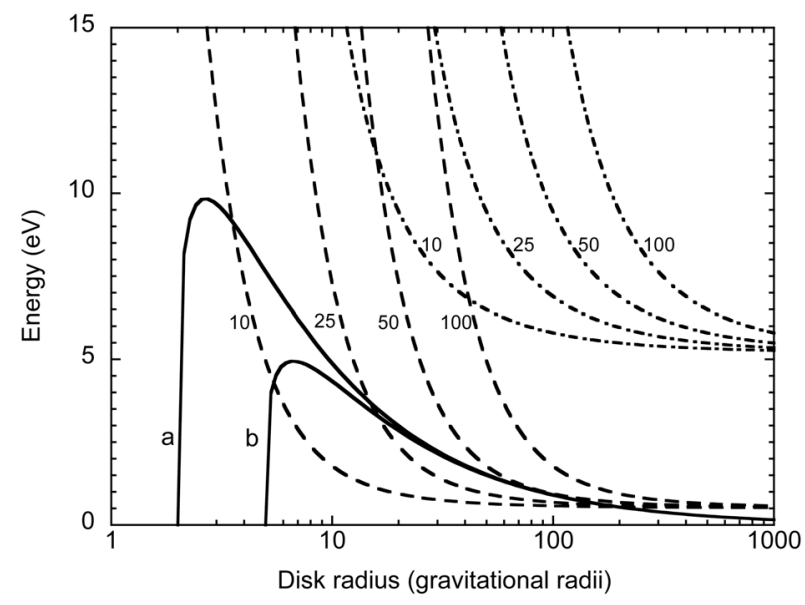

Fig. 4. Comparison of threshold energy and mean emitted energy for two accretion disk models. The mean energies of photons emitted by the disk at each radius are indicated by the solid curves. The curve labelled "a" represents the mean photon energy from a Shakura-Sunyaev accretion disk with parameters $M_{\mathrm{b}}=10^{9} M_{\odot}, \dot{m}=10^{-2}, r_{\mathrm{jet}} / r_{\mathrm{g}}=2$; the curve labeled " $b$ " represents the mean energy from a modified disk with the same parameters except that $r_{\text {jet }} / r_{\mathrm{g}}=5$. The dot-dash curves to the upper right show the threshold energy for disk photons emitted from a given radius interacting with $100 \mathrm{GeV}$ gamma-rays. The dashed curves represent the threshold energy for disk photons interacting with $1 \mathrm{TeV}$ gamma rays. The different threshold curves are derived from gamma-ray emission heights of $10,25,50$ and 100 gravitational radii respectively, as indicated on the curves.

for two accretion disk models. The first is a Shakura-Sunyaev model with black hole mass, $M_{\mathrm{bh}}=10^{9} M_{\odot}$, normalized accretion rate, $\dot{m}=10^{-2}$, no jet and $r_{\mathrm{ms}} / r_{\mathrm{g}}=2$; the second model has the same $M_{\mathrm{bh}}, \dot{m}$ and $r_{\mathrm{ms}}$ but has a jet with $r_{\text {jet }} / r_{\mathrm{g}}=5$. The decrease in disk emission and the deficit of high energy photons of the second model are apparent. We also show in this plot the threshold energy as a function of disk radius for photons interacting with (a) $1 \mathrm{TeV}$ and (b) $100 \mathrm{GeV}$ photons at heights of 10-100 gravitational radii. Clearly, the interaction of disk photons with $100 \mathrm{GeV}$ gamma-rays is almost insignificant irrespective of the particular disk model. However, it is clear from the comparison of threshold energies with the mean disk photon energy that there is substantial interaction between disk photons and $1 \mathrm{TeV}$ gamma-rays. However, the modified disk model decreases the interaction from photons emitted close to the jet since the emission is truncated for $r<5 r_{\mathrm{g}}$, the mean disk photon energy decreases substantially near $r=5 r_{\mathrm{g}}$ and the threshold energy also rises sharply in this region.

The comparison of mean emitted and threshold energy in Fig. 4 provides additional justification for the neglect of gravitational redshift. For heights of the gamma ray emitting region in excess of $25 r_{\mathrm{g}}$ the mean disk photon energy is less than the threshold inward of about $15 r_{\mathrm{g}}$ indicating a reduction in the contribution to the opacity of photons emitted close to the black hole. For gamma ray emission heights $\approx 10 r_{\mathrm{g}}$ from the black hole there will be some effect from the gravitational redshift. However, our estimates of opacity in this region are much better than "factor of order unity" and the trends discussed in the following section are robust.

\section{Relativistic effects}

We have used a Newtonian disk model, assuming that the details of disk emission external to $\approx 5 r_{\mathrm{g}}$ would not make a large difference to our estimates of opacity. In this section we estimate effects related to general relativity.

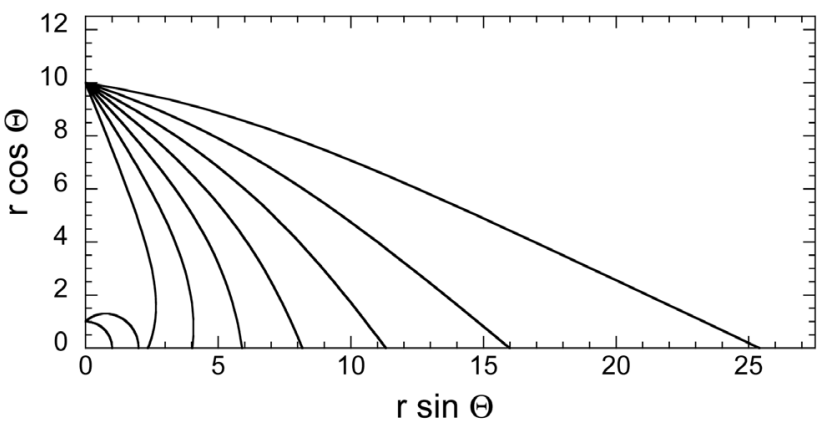

Fig. 5. Trajectories in Boyer-Lindquist coordinates of photons intercepting the symmetry axis of a maximally rotating black hole. The event horizon and static limit surface of the black hole are indicated.

There are three principal effects to consider: (1) the redshift of the emitted gamma rays. (2) The change in energy of a photon along its trajectory from the disk surface to the gamma ray emitting region along the axis of symmetry of the black hole. (3) The change in the locations of the footpoints (in the disk) of photons which intercept the symmetry axis. (4) The disk spectrum modified by relativistic effects. Here we consider the first three of these with (3) being particularly important.

We consider relativistic effects in the following way. We first consider the equations for particle trajectories in the Kerr metric given by Bardeen et al. (1972) specifically for the case of zero rest mass. Moroever, we consider photons which intersect the symmetry axis; these have zero angular momentum, $L$. The equations for the photon trajectories in Boyer-Lindquist coordinates, $(r, \Theta, \phi, t)$, where $r$ is in units of gravitational radii and $t$ is in units of $G M / c^{3}$ are:

$$
\begin{aligned}
& \Sigma \frac{\mathrm{d} r}{\mathrm{~d} \lambda}= \pm V_{r}^{1 / 2}(r) \\
& \Sigma \frac{\mathrm{d} \Theta}{\mathrm{d} \lambda}= \pm V_{\Theta}^{1 / 2}(\Theta) \\
& \Sigma \frac{\mathrm{d} \phi}{\mathrm{d} \lambda}=-2(a / m) \frac{r}{\Delta} \\
& \Sigma \frac{\mathrm{d} t}{\mathrm{~d} \lambda}=\frac{T^{2}}{\Delta}-(a / m) \sin ^{2} \Theta
\end{aligned}
$$

where

$$
\begin{aligned}
& \Sigma(r, \Theta)=r^{2}+(a / m)^{2} \cos ^{2} \Theta \\
& \Delta(r)=r^{2}-2 r+(a / m)^{2} \\
& T(r)=r^{2}+(a / m)^{2} \\
& V_{r}(r)=T^{2}(r)-\left[Q+(a / m)^{2}\right] \Delta(r) \\
& V_{\Theta}(\Theta)=Q+(a / m)^{2} \cos ^{2} \Theta .
\end{aligned}
$$

In order to determine the constant of the motion discovered by Carter (1968), which enters into the above equations, we consider photons which intercept the axis of symmetry at the location $r=r_{0}$ at the angle $\theta$ indicated in Fig. 1. In this case,

$Q\left(r_{0}, \theta\right)=\frac{T\left(r_{0}\right)^{2}}{\Delta\left(r_{0}\right)} \sin ^{2} \theta-(a / m)^{2}$.

We integrate the above equations backwards from the axis of symmetry until they either intercept the accretion disk $(\theta=\pi / 2)$ or the static limit surface at $r=1-\sqrt{1-(a / m)^{2} \cos ^{2} \theta}$. A typical set of trajectories for a maximally rotating black hole is shown in Fig. 5. In the figure it is apparent that photons are affected by the gravitational field: all trajectories beginning at the accretion disk curve downwards as they approach the vicinity of the black hole; 
trajectories beginning near the black hole (within about $5 r_{\mathrm{g}}$ ) and initially traveling outwards are pulled back towards the axis. The effect of this is to focus the photons propagating towards the axis, increasing the photon density there. Another way of looking at this is that the footpoints of photons intercepting the axis at a given angle are moved inwards compared to where they would be if there were no gravitational field. The relative movement is greatest for the trajectories which begin furthest out from the black hole. Relativistic effects will therefore increase pairabsorption.

In order to semi-quantitatively estimate the effect of the enhancement of photon density resulting from the gravitational field, we consider the integrand appearing in the expression (8) for the absorption length. The absorption cross-section peaks at $s \approx 2$ so that for a specific angle $\theta=\cos ^{-1} \mu$ the dominant contribution from the photon density occurs at an energy $\epsilon=4\left(m_{e} c^{2}\right)^{2} E^{-1}(1-\mu)^{-1}$, i.e. at twice the threshold energy. We have evaluated the photon density contribution at this energy using the Newtonian model adopted in this paper and also by taking into account the shifted footpoints of the photon trajectories discussed above but still using the Newtonian disk model. The disk temperature is higher at each shifted footpoint with a consequent shift on the blackbody spectrum. In addition, if $\epsilon$ is the photon energy on axis and $\epsilon_{0}$ is the the photon energy at the disk surface, then the photon density on axis is related to the photon density at the disk by:

$$
\frac{\mathrm{d} n}{\mathrm{~d} \epsilon \mathrm{d} \Omega}=\left(\frac{\epsilon}{\epsilon_{0}}\right)^{2} \frac{\mathrm{d} n}{\mathrm{~d} \epsilon_{0} \mathrm{~d} \Omega} .
$$

All energies are calculated in the local non-rotating frame (LNRF) of Bardeen (1970).

This procedure gives us an approximate idea of the differences between our Newtonian model and a fully relativistic treatment. As one expects the errors are largest for gamma ray emission regions closest to the black hole - as large as 30 percent for $z=10 r_{\mathrm{g}}$ and decreasing slowly for points at larger distances. At $z=100 r_{\mathrm{g}}$ the estimated error is about 15 percent; at $z=500 r_{\mathrm{g}}$ there is about a 5 percent effect. These estimates confirm that the optical depths calculated here are reasonably accurate and that the qualitative conclusions concerning the dependence of optical depth on distance from the black hole and the effect of the radiation field of the disk are robust.

\section{Discussion and conclusions}

For the parameters that we have used in this paper, we have shown that if the disk external to the jet in PKS 2155-304 dissipates a substantial portion of the gravitational power resulting from accretion in radiation, as indicated by our truncated Shakura \& Sunyaev (1973) model, then the pair opacity close to the black hole is significant at $\gtrsim \mathrm{TeV}$ gamma-ray energies and possibly lower, depending on the precise value of the normalized accretion rate. For a modest value of the normalised accretion rate, $\dot{m}=10^{-3}$ corresponding to a jet power $\approx 10^{43} \mathrm{erg} \mathrm{s}^{-1}$, the opaque region at an energy of $1 \mathrm{TeV}$ extends to approximately 400 gravitational radii $\approx 6 \times 10^{16} \mathrm{~cm}$. Within the region pair-absorption is expected to be detectable especially through spectral monitoring. The absence of spectral changes during outbursts provides constraints against even modest amounts of pairabsorption.

How does this conclusion depend upon parameters such as the jet radius, which we have not varied in this paper and also the assumption that the jet is Poynting flux dominated? The TeV optical depth is high for $r_{\text {jet }}=5 r_{\mathrm{g}}$ and $\dot{m} \gtrsim 10^{-3}$ and any reduction in jet radius would make the optical depth even larger since there would be more radiation illuminating the jet. An increase in jet radius decreases the ambient radiation density. However, even for $r_{\text {jet }}=10 r_{\mathrm{g}}$ the optical depth for energies of $10^{12}$ and $10^{12.5} \mathrm{eV}$ is unity at heights of 37 and $124 r_{\mathrm{g}}$ respectively. A jet radius of $10 r_{\mathrm{g}}$ is larger than is usually contemplated for jets in active galaxies. Hence our conclusion that for $\dot{m} \gtrsim 10^{-3}$ the very high energy gamma ray emission (say $E \gtrsim 10^{12} \mathrm{eV}$ ) is optically thick out to approximately 100 gravitational radii is secure.

The major source of uncertainty in the calculations is the spectrum of the accretion disk. This is not only influenced by relativistic effects but also by the effect of potential disk winds outside the jet region (Kuncic \& Bicknell 2007). We have assumed that the jet is disk-driven as proposed by Blandford \& Payne (1982) and investigated by numerous workers since. If the jet is driven by the process proposed by Blandford \& Znajek (1977), then in that case the disk exists at small radii (certainly within $5 r_{\mathrm{g}}$ ) and again the ambient radiation field exceeds what we have estimated here.

We have also investigated the alternative case in this paper, namely that the disk luminosity is well below that implied by the Shakura \& Sunyaev (1973) model. We have noted that this would be the case if a disk wind existed which advected energy away from the disk, reducing the dissipation of gravitational power. A comparison with M 87 is useful: taking into account the revised mass estimate of $M$ 87's black hole, $M_{\mathrm{bh}} \approx 6.4 \times$ $10^{9} M_{\odot}$ (Gebhardt \& Thomas 2009), the ratio of disk luminosity to Eddington luminosity is approximately $9 \times 10^{-7}$ (Whysong \& Antonucci 2004; Jolley \& Kuncic 2007). In the models we have used in this paper, the ratio of disk luminosity to Eddington luminosity is $\left(r_{\text {jet }} / r_{\mathrm{g}}\right) \dot{m}=0.2 \dot{m}$. For the lowest values of $\dot{m}=10^{-5}$, the ratio is comparable to that inferred for M 87. Thus there is good reason to suppose that the disk in PKS 2155-304 may not radiate at anything like the Shakura-Sunyaev rate and that the gamma ray emission originates from close to the accretion disk. For $\dot{m}=10^{-5}$ spectral changes at $1 \mathrm{TeV}$ would only be detectable if the emitting region is closer than $\approx 30 r_{\mathrm{g}}$.

Acknowledgements. We gratefully acknowledge support from the Go8-DAAD scheme which enabled this research to be carried out. We also acknowledge valuable support from Sonderforschungbereich 439.

\section{References}

Aharonian, F., Akhperjanian, A. G., Bazer-Bachi, A. R., et al. 2005, A\&A, 442, 895

Aharonian, F., Akhperjanian, A. G., Bazer-Bachi, A. R., et al. 2007, ApJ, 664, L71

Aharonian, F., Akhperjanian, A. G., Anton, G., et al. 2009, ApJ, 696, L150

Bardeen, J. M. 1970, ApJ, 162, 71

Bardeen, J. M., Press, W. H., \& Teukolsky, S. A. 1972, ApJ, 178, 347

Begelman, M. C., Fabian, A. C., \& Rees, M. J. 2008, MNRAS, 384, L19

Bicknell, G. V., \& Li, J. 2007, ApSS, 311, 275

Blandford, R. D., \& Levinson, A. 1995, ApJ, 441, 79

Blandford, R. D., \& Payne, D. G. 1982, MNRAS, 199, 883

Blandford, R. D., \& Znajek, R. L. 1977, MNRAS, 179, 433

Carter, B. 1968, Phys. Rev., 174, 1559

Donea, A.-C., \& Biermann, P. L. 1996, A\&A, 316, 43

Donea, A.-C., \& Protheroe, R. J. 2003, Astrop. Phys., 18, 377

Gebhardt, K., \& Thomas, J. 2009, ApJ, 700, 1690

Gould, R. J., \& Schréder, G. P. 1979, Phys. Rev., 155, 306

Haardt, F., \& Maraschi, L. 1991, ApJ, 380, L51

Haardt, F., \& Maraschi, L. 1993, ApJ, 413, 507

Haardt, F., Maraschi, L., \& Ghisellini, G. 1994, ApJ, 432, L95

Jolley, E. J. D., \& Kuncic, Z. 2007, ApSS, 311, 257

Kuncic, Z., \& Bicknell, G. V. 2004, ApJ, 616, 669

Kuncic, Z., \& Bicknell, G. V. 2007, Mod. Phys. Lett. A, 22, 1685

Shakura, N. I., \& Sunyaev, R. A. 1973, A\&A, 24, 337

Whysong, D., \& Antonucci, R. 2004, ApJ, 602, 116 